\title{
Electrolytic Lesions of the Fimbria/Fornix, Dorsal Hippocampus, or Entorhinal Cortex Produce Anterograde Deficits in Contextual Fear Conditioning in Rats
}

\author{
Stephen Maren*,1 and Michael S. Fanselow ${ }^{*}$ \\ *Department of Psychology, University of Michigan, 525 E. University Avenue, Ann Arbor, Michigan 48109-1109; and \\ †Department of Psychology, University of California, 405 Hilgard Avenue, Los Angeles, California 90095-1563
}

Recent data indicate that dorsal hippocampal (DH) lesions disrupt Pavlovian fear conditioning to contextual cues in rats. In the present study, we examined the effects of electrolytic lesions of the fimbria/fornix (FX) or entorhinal cortex (EC), the primary afferent projection systems to the $\mathrm{DH}$, on contextual fear conditioning in rats. Conditioning consisted of the delivery of unsignaled footshocks in a novel observation chamber, and freezing served as the measure of conditional fear. Electrolytic lesions of the $\mathrm{FX}, \mathrm{DH}$, or EC made 1 week before training produced anterograde impairments in both immediate postshock freezing on the conditioning day and freezing during the context extinction test $24 \mathrm{~h}$ following training. The deficits in conditional freezing produced by $\mathrm{FX}, \mathrm{DH}$, and EC lesions were not statistically different, although the deficits in rats with $\mathrm{FX}$ or EC lesions tended to be more severe than those in rats with $\mathrm{DH}$ lesions. In addition to producing deficits in conditional freezing, FX, DH, or EC lesions produced a pronounced locomotor hyperactivity. Within the lesion and sham groups, however, locomotor activity was not significantly correlated with conditional freezing. These results indicate that contextual fear deficits in rats with hippocampal formation damage are equivalent following either $F X$, $\mathrm{DH}$, or EC lesions. The relationship of freezing deficits and locomotor hyperactivity in rats with hippocampal formation lesions is discussed.

In Pavlovian fear conditioning, an aversive unconditional stimulus (US), usually a footshock, is paired with an initially neutral cue, such as a tone or a contextual conditional stimulus (CS). After a few

\footnotetext{
${ }^{1}$ To whom correspondence and reprint requests should be addressed at Department of Psychology, University of Michigan, 525 E. University Avenue, Ann Arbor, MI 48109-1109. Fax: (313) 763-7480. E-mail: maren@umich.edu. This work was supported by a grant from the NIMH (MH37986) to M.S.F.
}

pairings, the CS comes to evoke a conditional fear response (CR) that consists of a number of behavioral and autonomic adjustments, including freezing (i.e., somatomotor immobility) and increases in arterial blood pressure (e.g., Fanselow, 1994). Several recent reports indicate that the dorsal hippocampus (DH) plays an important role in Pavlovian fear conditioning in rats. Specifically, conditional freezing to contextual CSs is disrupted by electrolytic DH le sions made either before (Kim et al., 1993; Phillips \& LeDoux, 1992, 1994; Selden et al., 1991; Young et al., 1994) or shortly after (Kim \& Fanselow, 1992) fear conditioning. I mpairments in contextual fear occur when USs are unsignaled (Kim et al., 1993; but c.f. Phillips \& LeDoux, 1994) or signaled by a tone CS (Kim \& Fanselow, 1992; Phillips \& LeDoux, 1992, 1994; Selden et al., 1991). In contrast, fear to discrete CSs is apparently not affected by DH lesions (Kim \& Fanselow, 1992; Phillips \& LeDoux, 1992, 1994; Selden et al., 1991).

Recognizing the involvement of the $\mathrm{DH}$ in contextual fear conditioning, it is of considerable interest to determine the role of the major projection systems of the $\mathrm{DH}$ in this form of learning. The primary sources of input to the $\mathrm{DH}$ are the entorhinal cortex (EC) and the fimbria/fornix (FX). The EC is the primary cortical interface of the $\mathrm{DH}$ and is a component of the pathway by which multimodal sensory information reaches the DH (Swanson \& Cowan, 1977). As such, the EC may transmit information about polysensory contextual stimuli to the DH (Amaral \& Witter, 1989). In contrast, the $F X$ is the primary subcortical interface of the $\mathrm{DH}$ and has an important role in regulating electrical activity in the $\mathrm{DH}$, including theta rhythm (e.g., Andersen et al., 1979). Both the EC (Cho et al., 1993; Leonard et al., 1995; 
Meunier et al., 1993; Nagahara et al., 1995; Otto et al., 1991; Shenk \& Morris, 1985) and FX (Eichenbaum et al., 1990; Gaffan, 1994; Packard \& McGaugh, 1992; Sutherland \& Rodriguez, 1989; Walker \& Olton, 1984) have been implicated in many different learning and memory paradigms, and lesions of either structure, at least in rats, typically produce effects similar to DH lesions (e.g., Aggleton et al., 1992; J arrard et al., 1984; Olton et al., 1982; Yee \& Rawlins, 1994). That both structures are involved in contextual fear conditioning is suggested by studies indicating that long-term potentiation (LTP) in EC-DH projections (Maren et al., 1994a,c) and theta rhythm in the DH (Maren et al., 1994c) are correlated with contextual fear conditioning. Moreover, we have recently found that electrolytic EC lesions disrupt fear conditioning to contextual stimuli when shocks are signaled by a tone CS during training (Maren \& Fanselow, 1995; but c.f. Phillips \& LeDoux, 1995). Based on these results, one would expect that damage to either the EC of $F X$ should attenuate Pavlovian fear conditioning to contextual stimuli when shocks are unsignaled during training.

To assess this possibility, we have compared the effects of anterograde electrolytic lesions of the FX or EC with lesions of the DH on contextual fear conditioning in rats. Conditioning consisted of the delivery of unsignaled footshocks in a novel observation chamber, and freezing served as the measure of conditional fear. Because electrolytic lesions of structures in the hippocampal formation (HF) have been reported to increase locomotor activity under some conditions (an effect that may interact with freezing; Blanchard et al., 1977; Douglas \& I saacson, 1964; Roberts et al., 1962), we also examined the relationship between motor activity and conditional freezing in rats with HF lesions.

\section{METHODS}

Subjects. The subjects were 40 adult male LongEvans rats $(300-500 \mathrm{~g})$ born and reared in the Department of Psychology vivarium at the University of California, Los Angeles. After weaning, the rats were group housed in same-sex cohorts. At the be ginning of the experiment, the rats were individually housed in standard stainless-steel hanging cages on a 14:10-h light-dark cycle (lights on at 7:00 AM) and had free access to food and tap water. After individual housing, the rats were handled daily (10-20 s per rat) for 5 days to acclimate them to the experimenter.
Behavioral apparatus. Four identical observation chambers $(28 \times 21 \times 22 \mathrm{~cm}$; Lafayette Instrument Co., N orth Lafayette, IN) were used for both conditioning and contextual fear testing. The chambers were constructed from aluminum (sidewalls) and Plexiglas (rear wall, ceiling, and hinged front door). The chambers were situated in chests located in a brightly lit and isolated room. A videocamera placed in front of the observation chambers allowed each subject's behavior to be observed and recorded by an experimenter in an adjacent room. The floor of each chamber consisted of 18 stainless-steel rods (4 mm diameter) spaced $1.5 \mathrm{~cm}$ apart (center-to-center). The rods were wired to a shock generator and scrambler (Lafayette Instrument Co.) for the delivery of footshock USs. The chambers were cleaned with a 5\% ammonium hydroxide solution, and stainless-steel pans containing a thin film of the same solution were placed underneath the grid floors before rats were placed inside. Background noise (70 $\mathrm{dB}, \mathrm{A}$-scale) was supplied by ventilation fans in each chest and adjacent shock scramblers.

Surgery. The rats were randomly assigned to one of four groups receiving either sham surgery $(n=$ 16) or electrolytic lesions of the FX $(n=8), \mathrm{DH}(n$ $=8)$, or EC $(n=8)$. Rats were anesthetized with an intraperitoneal injection of Nembutal (sodium pentobarbital, $65 \mathrm{mg} / \mathrm{kg}$ body wt.) and mounted in a ster eotaxic apparatus (David Kopf I nstruments, Tujunga, CA). The scal $p$ was incised and retracted, and head position was adjusted to place bregma and lambda in the same horizontal plane. Small burr holes ( $2 \mathrm{~mm}$ diameter) were drilled in the skull for the placement of a stainless-steel el ectrode insulated with E poxylite except for $0.5 \mathrm{~mm}$ at the tip. Bilateral electrolytic lesions were made in the $\mathrm{FX}(1.3 \mathrm{~mm}$ posterior to bregma, $0.5 \mathrm{~mm}$ lateral to bregma, and $3.6 \mathrm{~mm}$ ventral to dura; $1.3 \mathrm{~mm}$ posterior to bregma, $1.2 \mathrm{~mm}$ lateral to bregma, and $3.8 \mathrm{~mm}$ ventral to dura), DH ( $2.8 \mathrm{~mm}$ posterior to bregma, $1.6 \mathrm{~mm}$ lateral to bregma, and $3.3 \mathrm{~mm}$ ventral to dura; $4.2 \mathrm{~mm}$ posterior to bregma, $2.6 \mathrm{~mm}$ lateral to bregma, and $3.0 \mathrm{~mm}$ ventral to dura), and EC ( $6.8 \mathrm{~mm}$ posterior to bregma, $5.0 \mathrm{~mm}$ lateral to bregma, and $7.0 \mathrm{~mm}$ ventral to dura; $8.0 \mathrm{~mm}$ posterior to bregma, 5.0 $\mathrm{mm}$ lateral to bregma, and $5.0 \mathrm{~mm}$ ventral to dura). Electrolytic lesions were made with anodal, constant direct current at each location (1.0 mA for $20 \mathrm{~s}$ for $\mathrm{DH}$ and EC lesions; $3.0 \mathrm{~mA}$ for $5 \mathrm{~s}$ for $\mathrm{FX}$ lesions). No el ectrodes were implanted during sham surgery. Following surgery, the incision was closed with stainless-steel wound dips, and the rats were allowed to recover on a heating pad before returning 
to their home cage. Three rats were excluded from the data analysis due to either death (one rat in the EC group) or illness (one rat each in the $\mathrm{SH}$ and $\mathrm{FX}$ groups) following surgery.

Procedure One week following surgery, the rats were placed in the conditioning chambers in squads of four rats; the chamber position was counterbalanced for each squad and group. The rats received three unsignaled footshocks ( $1 \mathrm{~s}, 0.5 \mathrm{~mA}$; 64-s intershock interval) $3 \mathrm{~min}$ after being placed in the chambers. Sixty-four seconds following the final shock, the rats were returned to their home cages.

Fear conditioning on the training day was assessed by measuring freezing (somatomotor immobility except that necessitated by breathing) during the 64-s period before the first tone- shock trial and the 64-s periods following each of the three shock trials (i.e., immediate postshock freezing). Freezing is an associative fear response evoked by stimuli associated with aversive consequences (Fanselow, 1986). Briefly, an observer who was blind to the experimental conditions scored each rat for freezing (behavioral immobility except for movement necessitated by respiration) every $8 \mathrm{~s}$ for a total of eight observations per animal per 64-s period. Ambulatory crossovers (defined as horizontal translocations of the rat's body from one side of the conditioning chamber to the other) were counted during the preshock period to examine the effects of HF lesions on locomotor activity. Twenty-four hours after training, fear conditioning to the context was assessed by returning the rats to the conditioning chambers and scoring freezing during an 8-min extinction test (a minute refers to a 64-s block), yielding a total of 64 observations per rat.

Histology. Histological verification of lesion location was performed after behavioral testing. Rats were perfused across the heart with $0.9 \%$ saline followed by $10 \%$ Formalin. After extraction from the skull, the brains were postfixed in $10 \%$ Formal in for 2 days and $10 \%$ Formalin/30\% sucrose until sectioning. Coronal sections ( $50 \mu \mathrm{M}$ thick, taken every $200 \mu \mathrm{M})$ were cut on a cryostat $\left(-16^{\circ} \mathrm{C}\right)$ and wetmounted on glass microscope slides with $70 \%$ ethanol. After drying, the sections were stained with $0.25 \%$ thionin to visualize neuronal cell bodies. Lesions were verified by visual inspection of the stained brain sections, and reconstructions of the lesions were made on rat brain atlas templates (Swanson, 1992).

Data analysis. For each test period, the freezing data were transformed to a percentage of total obser- vations, a probability estimate that is amenable to analysis with parametric statistics. These probability estimates of freezing were analyzed using analysis of variance (ANOVA). Planned comparisons in the form of Fisher LSD tests were performed following a significant omnibus F-ratio. All data are represented as means \pm the standard errors of the means (SEMs).

\section{RESULTS}

\section{Histology}

Histological reconstructions from representative rats in each experimental group are shown in Fig. 1. All of the rats in the FX group had complete lesions of the fimbria/fornix rostral tothe dorsal hippocampus. In general, damage to other brain structures in this group was limited, but included the neocortex overlying the fimbria/fornix, the rostral pole of the dorsal hippocampus, and septal nuclei in some rats. In contrast, rats in the DH group exhibited lesions localized throughout the rostral - caudal extent of the dorsal hippocampus. These lesions were typically complete, involving hippocampal area CA1, area CA3, and the dentate gyrus. Half of the rats exhibited lesions that extended caudally to include rostral aspects of the dorsal subiculum. DH lesions were not associated with damage to the fimbria/fornix rostral to the dorsal hippocampus. Extrahippocampal damage, when present, included minimal damage to the neocortex overlying the hippocampus. Rats in the EC group exhibited extensive damage to both the medial and lateral entorhinal cortex. In many cases, damage extended into the perirhinal cortex, particularly ventral to the rhinal fissure. This perirhinal cortical damage was restricted to the most caudal aspects of the structure. In addition to the entorhinal cortex, rats in the EC group exhibited damage to the ventral subiculum and caudal dentate gyrus. Typically, the lesions did not involve damage to any of the hippocampal subfields. With the exception of minimal dorsal hippocampal damage in the FX group, lesions in the FX, $\mathrm{DH}$, and EC groups were entirely non-overlapping.

\section{Behavior}

Electrolytic lesions of the hippocampal formation produced marked deficits in Pavlovian fear conditioning. As shown in Fig. 2A, lesions of the FX, DH, or EC produced substantial deficits in conditional freezing on the conditioning day (i.e., immediate postshock freezing). Compared to SH rats, rats with HF lesions exhibited impaired immediate postshock 

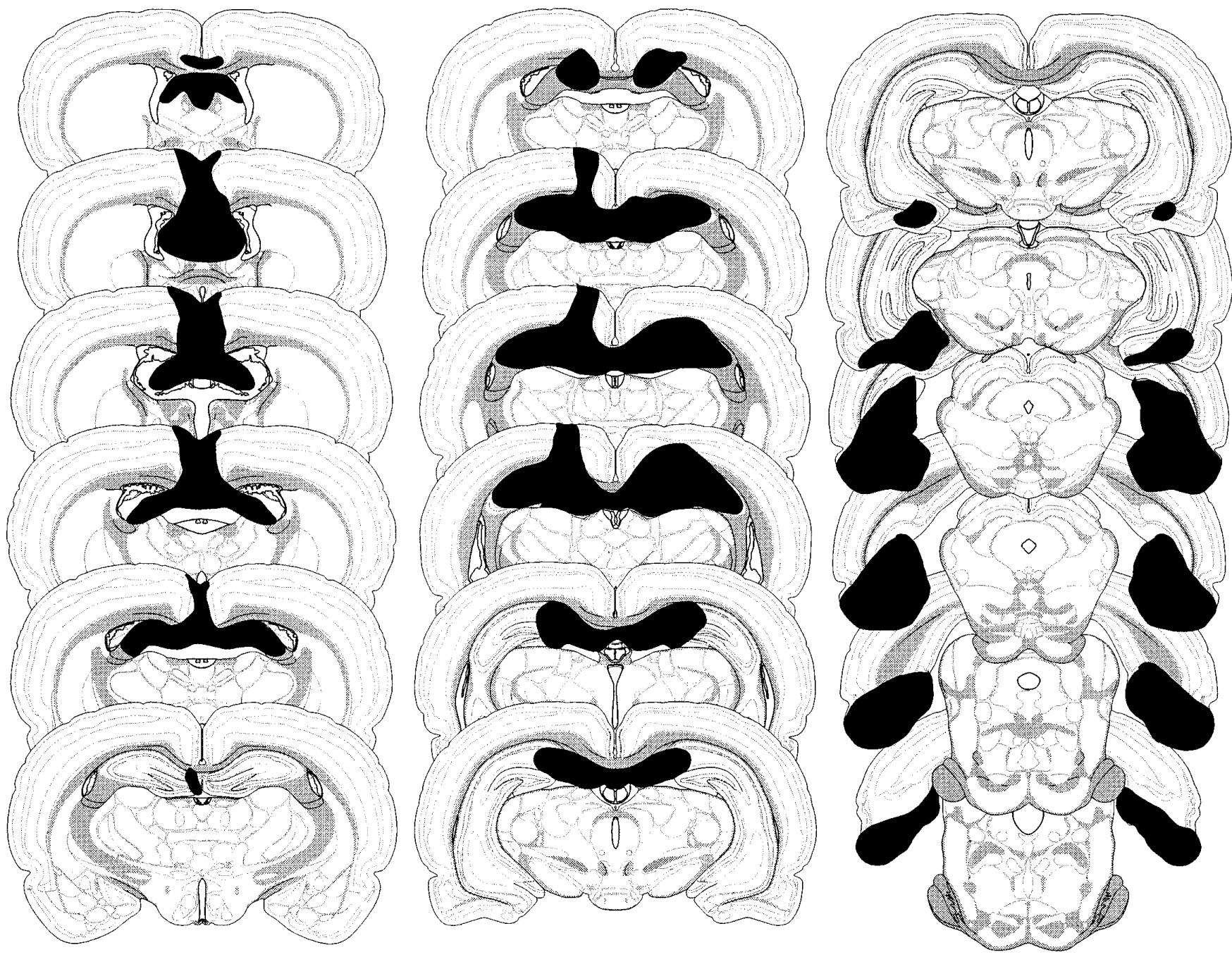

FIG. 1. Representative electrolytic lesions (black) of the fimbria/fornix (left column), dorsal hippocampus (center column), and entorhinal cortex (right column).

freezing at all postshock intervals; none of the groups differed in their levels of preshock (baseline) freezing. These observations were confirmed in an ANOVA, which revealed reliable main effects of group $[\mathrm{F}(3,33)=14.7, \mathrm{p}<.0001]$ and trial $[\mathrm{F}(3,99)$ $=114.9, \mathrm{p}<.0001]$ and a significant interaction between group and trial $[F(9,99)=3.9, p<.0005]$.

Deficits in conditional freezing in rats with $\mathrm{HF}$ lesions were not limited to the conditioning day. As shown in $\mathrm{Fig} .2 \mathrm{~B}$, rats with $\mathrm{FX}, \mathrm{DH}$, or $\mathrm{EC}$ lesions also exhibited impaired freezing during the 8-min context extinction test $24 \mathrm{~h}$ following training (data are collapsed across the 8-min test). This was confirmed by a significant main effect of group $[F(3,33)$ $=8.0, p<.0005]$ in the ANOVA. Planned comparisons $(p<.05)$ indicated that the $F X, D H$, and EC groups all differed from the SH group, but none of the HF groups differed from one another. Note, however, that although the planned comparisons did not reveal reliable differences between the HF groups, rats with $\mathrm{DH}$ lesions froze nearly twice as much as rats with $\mathrm{FX}$ or EC lesions. Thus, it is unlikely that the deficits in the $F X$ and EC groups are due to incidental hippocampal damage, because the group with the most hippocampal damage (i.e., the DH group) showed the weakest impairment in conditional freezing.

Figure $2 \mathrm{C}$ shows data from the 8-min context extinction test in 2-min blocks. The hippocampal groups were not significantly different from one another [ANOVA on FX, DH, and EC rats: group, $F(2$, $19)=1.9, p=.18$; group $x$ block, $F(6,57)=0.6, p$ $=.72 \mathrm{]}$ and were collapsed for clarity. As shown in Fig. 2C, the deficit in rats with HF lesions is ex- 

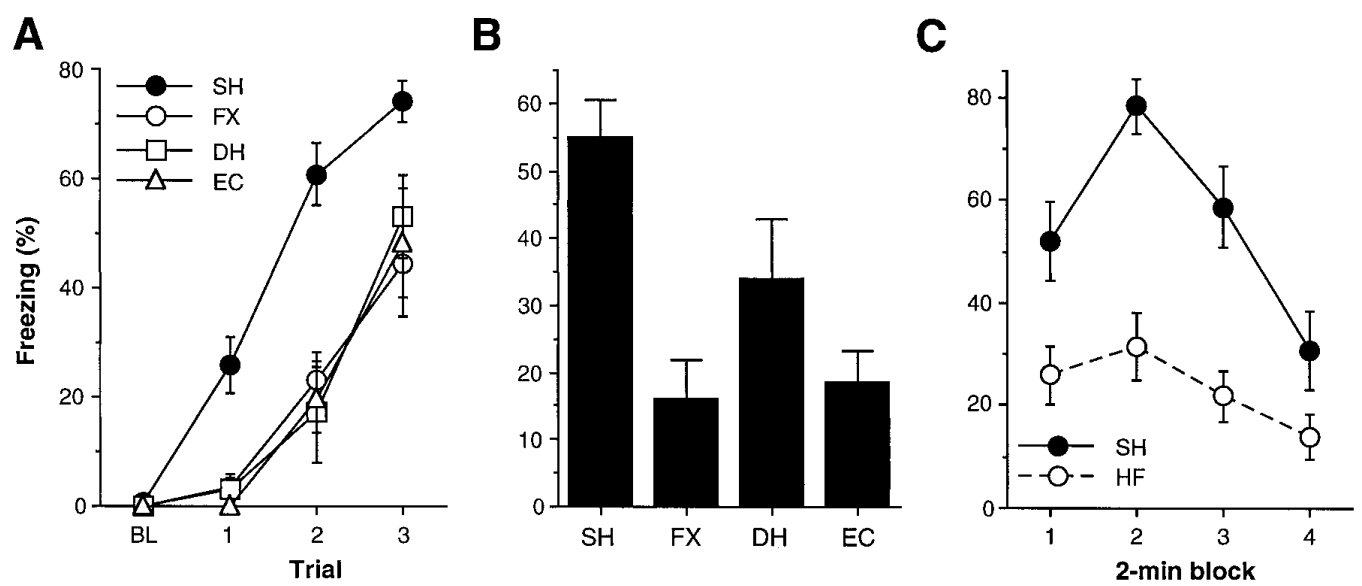

FIG. 2. Electrolytic lesions of the hippocampal formation and conditional freezing to contextual cues. (A) Mean ( \pm SEM) percentage of freezing on the conditioning day for rats in the sham ( $\mathrm{SH}$, filled circles), fornix (FX, open circles), dorsal hippocampus (DH, open squares), and entorhinal cortex (EC, open triangles) groups. Freezing was scored during 64-s intervals before (BL) and after each of the three footshock trials. Rats with electrolytic lesions of the FX, DH, or EC showed equivalent and significant deficits in immediate postshock freezing relative to rats receiving sham surgery. (B) Mean ( \pm SEM) percentage of freezing during the context extinction test $24 \mathrm{~h}$ following conditioning for rats in each of the groups described in (A). Data are collapsed across the 8-min extinction test. Similar to the immediate postshock freezing data, FX, DH, or EC lesions produced equivalent and significant deficits in conditional freezing to the context of the conditioning chamber. (C) Mean ( \pm SEM) percentage of freezing for each of the 2-min blocks of the context extinction test for rats in the groups described in (A). Data are collapsed across the three lesions groups to yield a hippocampal formation (HF) group. Rats with HF lesions (open circles) showed significantly lower conditional freezing to the training context relative to rats in the $\mathrm{SH}$ group (filled circles) across all $8 \mathrm{~min}$ of the extinction test.

pressed during every time point of the extinction test. This was confirmed by planned comparisons ( $p<.05$ ) following the significant group $\times$ block interaction in the ANOVA $[F(3,105)=4.3, p<.01]$. Thus, the freezing deficit in rats with HF lesions cannot be attributed to a rapid extinction of an initially high level of fear.

In addition to freezing, we quantified locomotory activity (ambulatory crossovers) during the 3-min preshock period on the conditioning day. It has been reported that HF lesions increase locomotor activity in some environments (Blanchard et al ., 1977; Douglas \& Isaacson, 1964; Mitchell et al., 1993; Roberts et al., 1962); hence we attempted to determine if $\mathrm{FX}, \mathrm{DH}$, or $\mathrm{EC}$ lesions increase locomotor activity in the conditioning boxes prior to footshock and, if so, the relationship of this activity to conditional freezing. Ambulatory crossovers during the 3-min preshock period are shown in Fig. 3A. As shown, FX, DH, and EC lesions all produced substantial increases in preshock crossovers. This was confirmed by a significant main effect of group $[F(3,33)=7.9, p<.0005]$ in the ANOVA. Planned comparisons $(p<.05)$ indicated that rats in the FX, DH, and EC groups differed from SH rats. In addition, FX rats showed reliably greater crossover activity than $\mathrm{DH}$ rats, but were not reliably different from rats with EC lesions; EC and DH rats were not different from one another. Thus, HF lesions increase locomotor activity in the same boxes that are used to assess conditional freezing.

The increase in preshock activity produced by HF lesions might compete with freezing behavior, thereby accounting for reduced freezing in rats with HF le sions. Indeed, Fig. 3B shows that the effect of HF lesions on crossovers covaries with that on conditional freezing. However, the two variables were not related in individual animals. In fact, the within-group Pearson correlations between crossovers and freezing in $\mathrm{SH}$ and HF rats (shown in Fig. 3C) are near 0. The within-group correlations for each hippocampal group also failed to achieve statistical significance ( $p s>.05$ ). The absence of within-group correlations between conditional freezing and preshock crossovers indicates that while crossover activity serves as a useful index of the overall increase in activity in hippocampal rats, it does not predict freezing in individual animals. Thus, it is not dear if the deficit in conditional freezing produced by HF lesions is caused by lesion-induced increases in locomotor activity or if increased locomotor activity and decreased conditional freezing are symptomatic of a common syndrome, such as a contextual learning deficit.

\section{DISCUSSION}

The present results indicate that electrolytic lesions of the $\mathrm{FX}, \mathrm{DH}$, or EC produce equivalent defi- 

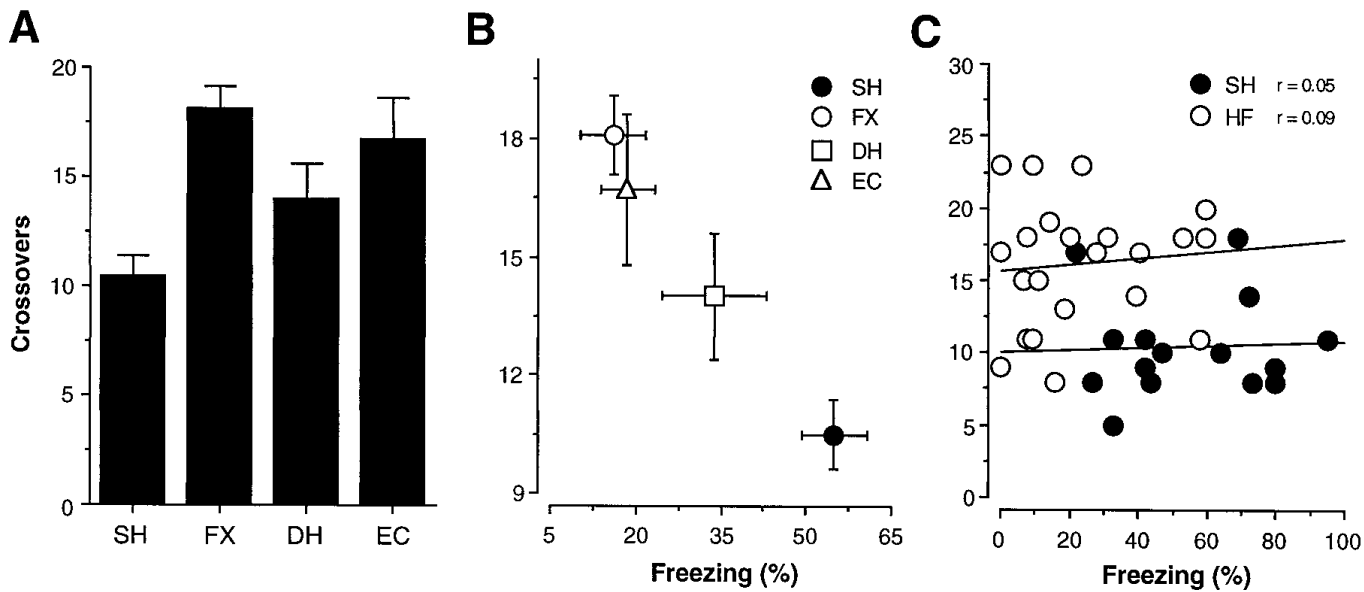

FIG. 3. Electrolytic lesions of the hippocampal formation and preshock locomotor activity. (A) Mean ( \pm SEM) ambulatory crossovers for rats in the SH, FX, DH, and EC groups. Crossovers were counted during the 3-min preshock period on the conditioning day, and the data are collapsed across this 3-min interval. Electrolytic lesions of the FX, DH, or EC reliably increased ambulatory crossovers compared to sham surgery. (B) Mean ( \pm SEM) ambulatory crossovers plotted against the mean $( \pm S E M)$ percentage of freezing during the 8-min context extinction test. Data indicate the relative efficacies of FX (open circles), DH (open squares), or EC (open triangles) lesions to both increase locomotor activity and decrease conditional freezing relative to SH (filled circles) rats. (C) Scatterplot of preshock ambulatory crossovers and conditional freezing during the extinction test for individual rats in the SH and HF (collapsed across FX, DH, and EC) groups. Pearson correlations (lines) for the SH (filled circles) and HF (open circles) groups (inset) were near 0.

cits in Pavlovian fear conditioning to contextual stimuli in rats. The deficits in freezing were apparent both on the conditioning day and during the delayed context extinction test. While rats in the $\mathrm{FX}$ and EC groups possessed some hippocampal damage, the freezing deficits in these rats were probably not the result of this damage, given that they froze less than rats in the DH group. The effects of electrolytic DH lesions reported here are consistent with those reported previously from this laboratory (e.g., Kim et al., 1993). Moreover, the comparable effects of $\mathrm{FX}, \mathrm{DH}$, and $\mathrm{EC}$ lesions are consistent with the equivalent effects of these lesions in other learning paradigms (e.g., Aggleton et al., 1992; J arrard et al., 1984; Olton et al., 1982; Yee \& Rawlins, 1994). Collectively, these data indicate an important role for both the cortical and subcortical interfaces of the DH in the acquisition of conditional freezing to a context.

While these results might be expected based on the effects of FX and EC lesions in other hippocampus-dependent learning paradigms, they are not entirely congruent with a recent report from LeDoux and colleagues (Phillips \& LeDoux, 1995). These investigators found that electrolytic $F X$ lesions, but not EC aspirations or combined EC and perirhinal cortex lesions, attenuated fear conditioning to contextual cues trained in the background to a tone CS. Phillips and LeDoux (1994) have also failed to find deficits in contextual fear conditioning in rats with electrolytic DH lesions when footshocks are unsignaled. These results may raise questions about the generality of these deficits. However, there are a number of differences between the present study and the Phillips and LeDoux (1994, 1995) studies that might account for the different results. For instance, the absence of an effect of $E C$ lesions in the Phillips and LeDoux (1995) study could be due either to the tone training procedure they used, their use of aspiration lesions, or the procedures they used to test contextual fear.

With regard to the first possibility, it is unlikely that the use of a tone CS is responsible for the lack of effect of $\mathrm{EC}$ lesions, because we have recently re ported that electrolytic EC lesions produce severe impairments in freezing to contextual cues trained in the background to a tone CS (Maren \& Fanselow, 1995). It may be the case that EC aspirations produce different behavioral effects than electrolytic EC lesions. In fact, there is precedent for this possibility as Douglas and Isaacson (1964) have reported that electrolytic and aspiration lesions of the $\mathrm{DH}$ produce different effects on spontaneous alternation, for example. Alternatively, the different procedures for testing conditional fear may have yielded the different results. Phillips and LeDoux $(1994,1995)$ score context freezing during one short 20-s interval each day for 2 days; we use a much longer 8-min test that may be more sensitive to small differences in freezing.

Another possibility is that LeDoux and colleagues expose all of their subjects to the conditioning chambers for $20 \mathrm{~min}$ on the day before conditioning. Inso- 
far as context conditioning deficits in hippocampal rats are due to failures in context representation (e.g., Kim \& Fanselow, 1992), the long context preexposure period used by Phillips and LeDoux (1994, 1995) may provide rats with EC or DH lesions ample time to form a context representation that can support conditioning when paired with footshock. Indeed, fear conditioning that is limited by the time allowed to establish a context representation can be substantially augmented by relatively short (2-min) preexposure periods (Fanselow, 1986, 1990). Alternatively, context preexposure may generate latent inhibition of contextual conditioning in control subjects, but not in DH or EC rats (Yee et al., 1995), which would have the effect of equalizing the levels of fear conditioning in the two groups. Thus, the procedures used by Phillips and LeDoux $(1994,1995)$ may not have afforded a strong test of the role of the HF in contextual fear conditioning, at least in a signaled shock situation.

In addition to reducing contextual freezing, we have found that HF lesions increase locomotor activity in the conditioning boxes prior to footshock. This is congruent with the large body of previously cited literature indicating increased motor activity in rats with damage to the HF. Given this pattern of results, we thought it possible that HF lesions produced deficits in freezing by increasing a competing response (i.e., locomotor activity). While there were no within-group correlations between freezing and preshock crossovers, the large between-group correlation of these measures suggests they are related. However, whether or not locomotor hyperactivity is the cause for reduced freezing in hippocampal rats is not clear. It is relevant to this issue that conditional freezing to discrete CSs is typically not affected by HF lesions, which reliably decrease freezing to context CSs in the same rats (Kim \& Fansel ow, 1992; Phillips \& LeDoux, 1992, 1994, 1995; Selden et al., 1991; but c.f. Maren \& Fanselow, 1995). This suggests that rats with HF lesions can freeze at high levels despite their increased activity levels.

What are the implications of the present results for contextual CS pathways to the hippocampus? While the present results indicate the importance of the EC and FX in contextual fear conditioning, it is not clear to what extent either or both of these structures serves as the critical relay of contextual information to the DH. It is unlikely that the $\mathrm{FX}$ conveys context information to the $\mathrm{DH}$ in view of the anatomy of this system. Nonetheless, the FX may have a role in regulating hippocampal representation of context. Thus, hippocampal theta rhythm is correlated with synaptic plasticity (i.e., long-term potentiation or LTP) in EC-DH projections, and manipulations that enhance theta rhythm al so enhance EC-DH LTP and contextual fear conditioning (Maren et al., 1994b,c). It seems more likely that the EC serves as the critical relay for contextual information to the DH. Thus, deficits in fear conditioning following $\mathrm{EC}$ lesions may be the result of removing polymodal sensory input to the $\mathrm{DH}$, which is required for processing contexts. Alternatively, impairments in fear conditioning following EC lesions may result from eliminating contextual information flow from the EC to the basolateral amygdala (BLA). It is well known that the amygdaloid complex is essential for fear conditioning (Davis, 1992; LeDoux, 1995; Maren and F anselow, 1996), and we and others have suggested that EC-BLA projections are required for contextual fear conditioning ( $M$ aren and Fanselow, 1995, 1996; Phillips and LeDoux, 1992). Nonetheless, the relative contribution of EC-BLA projections compared to EC-DH projections to contextual fear conditioning remains to be determined.

\section{REFERENCES}

Aggleton, J . P., Keith, A. B., Rawlins, J . N., Hunt, P. R., \& Sahgal, A. (1992). Removal of the hippocampus and transection of the fornix produce comparable deficits on delayed non-matching to position by rats. Behavioural Brain Research, 52, 6171.

Amaral, D. G., \& Witter, M. P. (1989). The three-dimensional organization of the hippocampal formation: A review of anatomical data. Neuroscience, 31, 571-591.

Andersen, P., Bland, H. B., Myhrer, T., \& Schwartzkroin, P. A. (1979). Septo-hippocampal pathway necessary for dentate theta production. Brain Research, 165, 13- 22.

Blanchard, D. C., Blanchard, R. J ., Lee, E. M. C., \& Fukunaga, K. K. (1977). Movement arrest and the hippocampus. Physiological Psychology, 5, 331- 335.

Cho, Y. H., Beracochea, D., \& J affard, R. (1993). Extended temporal gradient for the retrograde and anterograde amnesia produced by ibotenate entorhinal cortex lesions in mice. J ournal of Neuroscience, 13, 1759- 1766.

Davis, M. (1992). The role of the amygdala in fear and anxiety. Annual Review of Neuroscience, 15, 353-375.

Douglas, R. J ., \& I saacson, R. L. (1964). Hippocampal lesions and activity. Psychonomic Science, 1, 187- 188.

Eichenbaum, H., Stewart, C., \& Morris, R. G. (1990). Hippocampal representation in place learning. J ournal of Neuroscience, 10, 3531- 3542.

Fanselow, M. S. (1986). Associative vs topographical accounts of the immediate shock-freezing deficit in rats: I mplications for the response selection rules governing species-specific defensive reactions. Learning and Motivation, 17, 16- 39.

Fanselow, M. S. (1990). Factors governing one-trial contextual conditioning. Animal Learning and Behavior, 18, 264- 270.

Fanselow, M. S. (1994). Neural organization of the defensive behavior system responsible for fear. Psychonomic Bulletin and Review, 1, 429- 438. 
Gaffan, D. (1994). Dissociated effects of perirhinal cortex ablation, fornix transection and amygdalectomy: Evidence for multiple memory systems in the primate temporal lobe. Experimental Brain Research, 99, 411- 22.

J arrard, L. E., Okaichi, H., Steward, O., \& Goldschmidt, R. B. (1984). On the role of hippocampal connections in the performance of place and cue tasks: Comparisons with damage to hippocampus. Behavioral Neuroscience, 98, 946-954.

Kim, J . J ., \& Fanselow, M. S. (1992). Modality-specific retrograde amnesia of fear. Science, 256, 675-677.

Kim, J . J ., Rison, R. A., \& Fanselow, M. S. (1993). Effects of amygdala, hippocampus, and periaqueductal gray lesions on shortand long-term contextual fear. Behavioral Neuroscience, 107, 1093- 1098.

LeDoux, J . E. (1995). Emotion: Clues from the brain. Annual Re view of Psychology, 46, 209- 235.

Leonard, B. W., Amaral, D. G., Squire, L. R., \& Zola-Morgan, S. (1995). Transient memory impairment in monkeys with bilateral lesions of the entorhinal cortex. J ournal of Neuroscience, 15, 5637-5639.

Maren, S., De Oca, B., \& Fanselow, M. S. (1994a). Sex differences in hippocampal long-term potentiation (LTP) and Pavlovian fear conditioning in rats: Positive correlation between LTP and contextual learning. Brain Research, 661, 25- 34.

Maren, S., DeCola, J . P., \& Fanselow, M. S. (1994b). Water deprivation enhances fear conditioning to contextual, but not discrete, conditional stimuli in rats. Behavioral Neuroscience, 108, 645-649.

Maren, S., DeCola, J . P., Swain, R. A., Fanselow, M. S., \& Thompson, R. F. (1994c). Parallel augmentation of hippocampal long-term potentiation, theta rhythm, and contextual fear conditioning in water-deprived rats. Behavioral Neuroscience, 108, 44- 56.

Maren, S., \& Fanselow, M. S. (1995). Synaptic plasticity in the basolateral amygdala induced by hippocampal formation stimulation in vivo. J ournal of Neuroscience, 15, 7548- 7564.

Maren, S., \& Fanselow, M. S. (1996). The amygdala and fear conditioning: Has the nut been cracked? Neuron, 16, 237- 240.

Meunier, M., Bachevalier, J., Mishkin, M., \& Murray, E.A. (1993). Effects on visual recognition of combined and separate ablations of the entorhinal and perirhinal cortex in rhesus monkeys. J ournal of Neuroscience, 13, 5418-5432.

Mitchell, D., Maren, S., \& Hwang, R. (1993). The effects of hippocampal lesions on two neotic choice tasks. Psychobiology, 21, 193- 202.

Nagahara, A. H., Otto, T., \& Gallagher, M. (1995). Entorhinalperirhinal lesions impair performance of rats on two versions of place learning in the Morris water maze. Behavioral Neuroscience, 109, 3-9.

Olton, D. S., Walker, J . A., \& Wolf, W. A. (1982). A disconnection analysis of hippocampal function. Brain Research, 233, 241253.

Otto, T., Schottler, F., Staubli, U., Eichenbaum, H., \& Lynch, G. (1991). Hippocampus and olfactory discrimination learning:
Effects of entorhinal cortex lesions on olfactory learning and memory in a successive-cue, go-no-go task. Behavioral Neuroscience, 105, 111- 119.

Packard, M. G., \& McGaugh, J . L. (1992). Double dissociation of fornix and caudate nucleus lesions on acquisition of two water maze tasks: Further evidence for multiple memory systems. Behavioral Neuroscience, 106, 439- 446.

Phillips, R. G., \& LeDoux, J. E. (1992). Differential contribution of amygdala and hippocampus to cued and contextual fear conditioning. Behavioral Neuroscience, 106, 274- 285.

Phillips, R. G., \& LeDoux, J . E. (1994). Lesions of the dorsal hippocampal formation interfere with background by not foreground contextual fear conditioning. Learning and Memory, 1, 34- 44.

Phillips, R. G., \& LeDoux, J. E. (1995). Lesions of the fornix but not the entorhinal or perirhinal cortex interfere with contextual fear conditioning. J ournal of Neuroscience, 15, 53085315.

Roberts, W. W., Dember, W. N., \& Brodwick, M. (1962). Alternation and exploration in rats with hippocampal lesions. J ournal of Comparative and Physiological Psychology, 55, 695700.

Selden, N. R., Everitt, B. J ., J arrard, L.E., \& Robbins, T.W. (1991). Complementary roles for the amygdala and hippocampus in aversive conditioning to explicit and contextual cues. Neuroscience, 42, 335- 350.

Shenk, F., \& Morris, R. G. M. (1985). Dissociation between components of spatial memory in rats after recovery from the effects of retrohippocampal lesions. Experimental Brain Research, 58, $11-28$.

Sutherland, R. J ., \& Rodriguez, A. J . (1989). The role of the fornix/ fimbria and some related subcortical structures in place learning and memory. Behavioural Brain Research, 32, 265277.

Swanson, L. W. (1992). Brain maps: Structure of the rat brain. New York: Elsevier.

Swanson, L. W., \& Cowan, W. M. (1977). An autoradiographic study of the organization of the hippocampal formation in the rat. J ournal of Comparative Neurology, 172, 49-84.

Walker, J . A., \& Olton, D. S. (1984). Fimbria-fornix lesions impair spatial working memory but not cognitive mapping. Behavioral Neuroscience, 98, 226- 242.

Yee, B. K., Feldon, J ., \& Rawlins, J . N. P. (1995). Latent inhibition in rats is abolished by NMDA-induced neuronal loss in the retrohippocampal region, but this lesion effect can be pre vented by systemic haloperidol treatment. Behavioral Neuroscience, 109, 227- 240.

Yee, B. K., \& Rawlins, J. N. (1994). The effects of hippocampal formation ablation or fimbria-fornix section on performance of a nonspatial radial arm maze task by rats. J ournal of Neuroscience, 14, 3766- 3774.

Young, S. L., Bohenek, D., \& Fanselow, M. S. (1994). NMDA processes mediate anterograde amnesia of contextual fear conditioning induced by hippocampal damage: Immunization against amnesia by context preexposure. Behavioral Neuroscience, 108, 19-29. 